\title{
Editorial
}

Herz $2020 \cdot 45: 1-2$

https://doi.org/10.1007/s00059-019-04889-9

(c) Springer Medizin Verlag GmbH, ein Teil von Springer Nature 2020

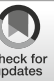

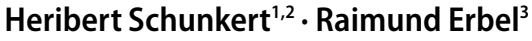

${ }^{\prime}$ Klinik für Herz- und Kreislauferkrankungen, Deutsches Herzzentrum München, Technische Universität München, München, Deutschland

${ }^{2}$ partner site Munich Heart Alliance, Deutsches Zentrum für Herz-Kreislauf-Forschung (DZHK) e.V., München, Deutschland

${ }^{3}$ Institut für Medizinische Informatik, Biometrie und Epidemiologie (IMIBE), Universitätsklinikum Essen (AöR), Essen, Deutschland

\section{Evidenzbasierte Primärprävention}

\section{Wo stehen wir im Jahr 2020?}

Wohl getan ist es, die Gesunden sorgfältig zu führen, damit sie nicht krank werden. (Hippokrates von Kos, 460-377 v. Chr., griechischer Arzt)

Die Herausforderungen in der Medizin haben sich wohl in den letzten 2500 Jahren wenig geändert. Schon damals erforderte es einen ärztlichen Ratgeber, um die Gesunden zu führen. Auch haben sich die Ratschläge wenig geändert. So fuhr Hippokrates fort: „Wer stark, gesund und jung bleiben will, sei mäßig, übe den Kör- per, atme reine Luft und heile sein Weh eher durch Fasten als durch Medikamente." Zwar sind wir nicht Zeitzeugen im alten Griechenland gewesen, allerdings vermuten wir, dass der heutige Lebensstil noch stärker von den Idealen des Hippokrates abweicht. Deutlich gewachsen sind dagegen unsere Kenntnisse über die Biologie und Ätiologie von chronischen, degenerativen Erkrankungen. In diesem Schwerpunktheft von Herz werden daher die wesentlichen diagnostischen und therapeutischen Implikationen einer evi- denzgestützten Primärprävention aufgezeigt.

Degenerative kardiovaskuläre $\mathrm{Er}$ krankungen entstehen in einem Wechselspiel aus Faktoren, die einerseits genetisch determiniert sind und andererseits durch unseren Lebensstil beeinflusst werden können. Entsprechend können diagnostisch zunächst genetische und familienanamnestische Informationen über das Risiko einer kardiovaskulären Erkrankung Auskunft geben. Im weiteren Lebensverlauf können Biomarker,

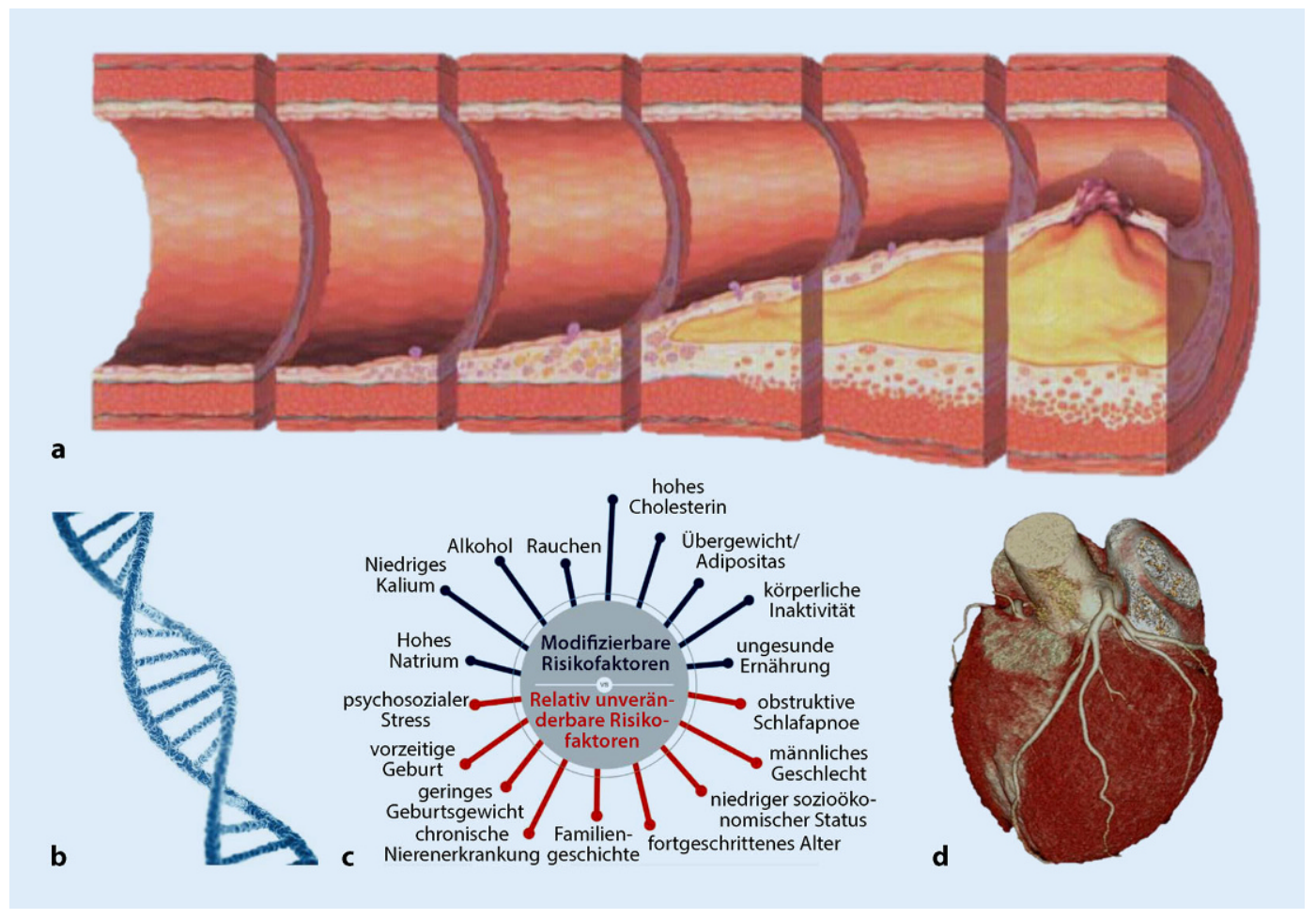

Abb. $1 \triangleleft$ Welche Art von Diagnostik zum Tragen kommt, hängt vom Stadium der degenerativen kardiovaskulären Erkrankungen (a) ab (b Genetik, c Genetik/Risikofaktoren/ Biomarker, d Bildgebung). (Gefäß: @ Wikipedia, Endo dysfunction Athero, CC BY-SA 3.0; DNA: (c) BlackJack3D/Getty Images/iStock; Risikofaktoren: präsentiert beim Jashreskongress der American Heart Association [AHA] 2019; Bildgebung: (C) H. Schunkert/Deutsches Herzzentrum München [DHM]) 
die diskrete Krankheitsmanifestationen im Blut widerspiegeln, diagnostisch erfasst und genutzt werden. Biomarker und Risikofaktoren können dann zusammengefasst in Scores abgebildet werden, die Auskunft über das langfristige Risiko geben. Kommt es zur Krankheitsmanifestation am Gefäßsystem, ist die moderne Bildgebung die aussagekräftigste Diagnostik. • Abb. 1 zeigt, in welcher Phase die Domäne der jeweiligen diagnostischen Instrumente liegt.

In diesem Heft finden Sie entsprechend Übersichtsartikel, die den aktuellen Stand zur genetischen Diagnostik, zu den modernen Risikoscores und zur Bildgebung abbilden. Wir sind glücklich, dass wir zu den jeweiligen Themenkomplexen hochkompetente Autoren finden konnten.

Unverändert ist seit Hippokrates, dass eine sinnvolle Therapie zur Primärprävention die größte Herausforderung darstellt. Welche Allgemeinmaßnahmen nutzbringend sind, haben wir für Kinder und Erwachsene untergliedert, denen wir jeweils ein eigenes Kapitel in diesem Heft gewidmet haben. Darüber hinaus ergibt sich eine Vielzahl von Möglichkeiten zur medikamentösen Primärprävention. Auf diesem Sektor hat sich seit Hippokrates vielleicht am meisten verändert. Unverändert ist jedoch die große Herausforderung, diese präventiven Maßnahmen, sei es in Form von Lebensstilmaßnahmen oder einer gezielten medikamentösen Behandlung, dem Patienten zu vermitteln. Auch diesbezüglich finden Sie viele Anregungen in diesem Heft.

Wir sind uns sicher, dass Hippokrates am Lesen dieses Heftes viel Freude gefunden hätte. Ich hoffe, Sie teilen diese Begeisterung für die Prävention von Gefäß- und Herzerkrankungen.

Mit freundlichen Grüßen

Ihre

Heribert Schunkert und Raimund Erbel

\section{Korrespondenzadresse}

Prof. Dr. med. Heribert Schunkert

Klinik für Herz- und Kreislauferkrankungen, Deutsches Herzzentrum München, Technische Universität München

Lazarettstr. 36, 80636 München, Deutschland schunkert@dhm.mhn.de

Interessenkonflikt. H. Schunkert und R. Erbel geben an, dass kein Interessenkonflikt besteht.

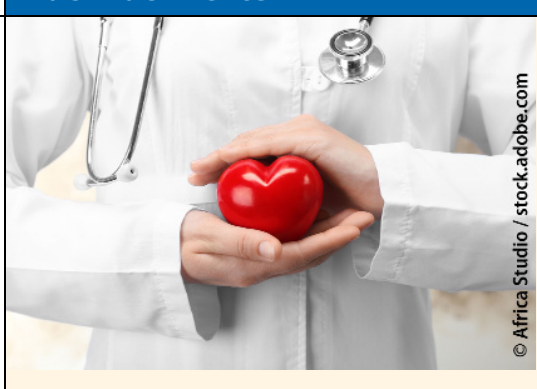

Für starke Frauenherzen

Mehr Frauen als Männer werden wegen einer Herzschwäche im Krankenhaus behandelt und sterben daran. Oft unterschätzen sie die Anzeichen und suchen viel später als Männer ärztliche Hilfe auf. Bei dieser Erkrankung des Herzens reicht die Pumpleistung nicht mehr aus, um den Körper ausreichend mit Sauerstoff zu versorgen. Die häufigsten Gründe sind Koronare Herzkrankheit, Bluthochdruck, Herzrhythmusstörungen, Herzmuskelentzündungen und Herzklappenfehler. Bei Frauen gehen der Herzschwäche häufig ein zu hoher Blutdruck, Erkrankungen der Herzklappen oder auch Diabetes voraus. Symptome wie Atemnot, Müdigkeit oder mögliche Herzrhythmusstörungen sind bei ihnen initial schwächer ausgeprägt als bei Männern oder werden häufig nicht als Warnsignal interpretiert. Dagegen leiden Frauen häufiger unter möglichen Begleiterscheinungen wie Ängsten oder Depressionen.

Mit einer aktiven, bewussten Lebensweise können Frauen dazu beitragen, Herz und Gefäße gesund zu erhalten und ihr Erkrankungsrisiko zu senken. Unter www.frauengesundheitsportal.de liefert das Frauengesundheitsportal Tipps für mehr Bewegung im Alltag, leicht umsetzbare Ideen für eine herzgesunde Ernährung und wirkungsvolle Strategien zum Abbau von Stress.

Darüber hinaus gibt das Portal einen Überblick zu weiteren Herz-Kreislauf-Erkrankungen wie Bluthochdruck, chronischem Herzversagen oder Herzrhythmusstörungen.

Quelle: Bundeszentrale für gesundheitliche Aufklärung (BZgA), www.bzga.de 